\title{
HIV-1 Tat protein induces the production of IDO in human monocyte derived-dendritic cells through a direct mechanism: effect on T cells proliferation
}

\author{
Rémi Planes ${ }^{1,2^{*}}$, Elmostafa Bahraoui ${ }^{1,2}$ \\ From Frontiers of Retrovirology: Complex retroviruses, retroelements and their hosts \\ Cambridge, UK. 16-18 September 2013
}

\section{Background}

During HIV-1 infection, an increase of indoleamine 2,3 dioxygenase (IDO) expression, and dendritic cells (DC) dysfunction were often associated with AIDS disease progression [1].

\section{Materials and methods}

In this work, we investigated the effect of Tat recombinant protein from HIV-1 Lai and SF-2 strains on the expression of IDO, in Monocyte-derived dendritic cells (MoDCs) generated following 5 days of culture in the presence of GM-CSF and IL-4. IDO expression was analysed by SDS-PAGE and western blotting, intracellular labelling or by measuring kynurenine production by Ehrlich's assay. The capacity of Tat-treated MoDC to stimulate $\mathrm{T}$ cell proliferation was analysed by following CFSE dilution in the presence or absence of IDO inhibitor (1-methyl-tryptophane).

\section{Results}

We show that Tat induces IDO protein expression and activity in a dose dependent manner by acting at the cell membrane level. Using different Tat-fragments, we show that the N-Terminal domain, Tat 1-45, but not the central region, Tat 30-72, is sufficient to induce the expression of active IDO. Tat protein is also able to induce several cytokines in MoDCs, including IFN- $\gamma$, a strong inducer of IDO. In order to understand whether IDO is induced directly by Tat protein or indirectly following IFN- $\gamma$ production, complementary experiments were performed and showed that: i) at the kinetic level, Tat induced IDO expression before the production of

${ }^{1}$ Université Paul Sabatier, 118 Route de Narbonne, Toulouse, France Full list of author information is available at the end of the article
IFN- $\gamma$ ii) treatment of MoDCs with Tat-conditioned medium was unable to stimulate IDO expression, iii) coculture of MoDCs in a transwell cell system did not allow IDO expression in MoDCs not previously treated by Tat, iv) direct contact between Tat-treated and untreated MoDCs was not sufficient to induce IDO expression in a Tat-independent manner, and v) treatment of MoDCs in the presence of IFN- $\gamma$ pathway inhibitors, Jak I and Ly294002, inhibited IFN- $\gamma$-induced IDO but had no effect on Tat-induced IDO. At the functional level, our data showed that treatment of MoDCs with Tat led to the inhibition of their capacity to stimulate $\mathrm{T}$ cell proliferation. This impairement was totally abolished when the stimulation was performed in the presence of $1 \mathrm{MT}$, an inhibitor of IDO activity, arguing for the implication of the kynurenine pathway.

\section{Conclusions}

By inducing IDO, Tat protein may be considered, as a viral pathogenic factor, in the dysregulation of the DC functions during HIV-1 infection.

\section{Authors' details}

${ }^{1}$ Université Paul Sabatier, 118 Route de Narbonne, Toulouse, France. ${ }^{2}$ INSERM, U1043, CPTP, CHU Purpan, Toulouse, France.

Published: 11 October 2013

\section{Reference}

1. Favre D, Mold J, Hunt PW, Kanwar B, Loke P, et al: Tryptophan catabolism by indoleamine 2,3-dioxygenase 1 alters the balance of $\mathrm{TH} 17$ to regulatory T cells in HIV disease. Sci Transl Med 2:32ra36.

doi:10.1186/1742-4690-10-S1-P113

Cite this article as: Planes and Bahraoui: HIV-1 Tat protein induces the production of IDO in human monocyte derived-dendritic cells through a direct mechanism: effect on T cells proliferation. Retrovirology 2013 10(Suppl 1):P113. 